\title{
Sistema de Apoio à Decisão Aplicado ao Planejamento e Distribuição da Coleta Seletiva de Resíduos Sólidos
}

\author{
Eugênio de Oliveira Simonetto ${ }^{1,2}$, Denis Borenstein ${ }^{2}$
}

${ }^{1}$ Curso de Sistemas de Informação - Centro Universitário Franciscano (UNIFRA) Andradas, 1614 - 97015-032 - Santa Maria - RS - Brasil

${ }^{2}$ Escola de Administração-Universidade Federal do Rio Grande do Sul (UFRGS) Rua Washington Luiz, 855 - 90010-460 - Porto Alegre-RS-Brasil

\footnotetext{
eosimonettoeunifra.br, denisbeea.ufrgs.br
}

Resumo. () artigo apresenta um sistema de apoio à decisão aplicado ao planejamento operacional da coleta seletiva de resíduos sólidos (SCOLIDSS), o qual tem por funcionalidade principal à geração de alternativas ao processo decisório no que se refere á: (a) alocação de veículos para a coleta seletiva, bem como o roteiro a ser percorrido pelos mesmos e, (b) a determinação da quantidade diária de resíduos sólidos a ser enviado a cada unidade de triagem. Para o desenvolvimento do mesmo foi utilizada a combinação de técnicas advindas da Pesquisa Operacional, que são a simulação computacional de eventos discretos e algoritmos para o problema da alocação e roteamento de vé́culos. O sistema foi desenvolvido utilizando o ambiente Borland Delphi e, para a simulação foi utilizado o simulador Arena 3.5. Para a validação do SCOLDSS estão sendo utilizados dados da coleta seletiva de um município do Rio Grande do Sul.

Abstract. The paper presents a decision support system (DSS) for recyclable waste collection planning. The computer system has the following objectives: (a) to define the vehicles' allocation and routing; (b) to determine the quantity of solid waste to be sent to each waste recyclable trial unit; and (c) to generate operational scenarios to be taken into account in the decision process. To accomplish such objectives the decision support system employs two wellknown operations research techniques, namely simulation, and assignment/VRP algorithms. The DSS was implemented in Borland Delphi, using the commercial package Arena 3.5 to carry out the simulations. The system was validated using a field test in one city of Rio Grande do Sul.

1. Introdução

A reciclagem dos resíduos sólidos é uma excelente alternativa para propiciar a preservação de recursos naturais, a economia de energia, redução do material que demanda o aterro sanitário, geração de emprego e renda, conscientização da população para questões ambientais. Porém, para um melhor funcionamento é de vital importância que se implante nas cidades um amplo sistema de coleta seletiva, onde os recicláveis e reutilizáveis sejam separados nas residências e coletados pelo sistema municipal de coleta seletiva. Apesar de ser uma excelente alternativa para a redução de resíduos com 
destino aos aterros, apenas $4 \%$ dos resíduos são reutilizados ou reciclados nas cidade gaúchas, segundo o CEMPRE (ONG-Compromisso Empresarial para a Reciclagen). Um dos motivos desta parcela reduzida de reciclagem deve-se ao nou acondicionamento dos resíduos pela população, fato este gerado pela falta de informação acerca da coleta seletiva. Outros fatores que contribuem para o pequeno índice de reciclagem dos resíduos é o alto custo da coleta seletiva para municipalidades (O'Leary, 1999; Monteiro, 2001) e a falta de um projeto para dimensionar de forma adequada à capacidade de armazenamento e processamento de resíduos nas unidades de triagem.

Além do alto custo da coleta, um fator que contribui bastante para os baixos índices de reciclagem é a falta de atenção dada à capacidade de armazenamento e processamento de resíduos nas unidades de triagem. Muitas vezes, os sistemas de coleta consideram, para a definição da distribuição dos resíduos, somente as distâncias ou os custos de deslocamentos dos pontos de coleta aos depósitos finais (Chang, 2000; Huang, 1998). Tais modelos, devido às características das disposições finais estudadas pelos mesmos, não levam em consideração o fluxo dinâmico de resíduos (entrada / saída) que possuem as unidades de triagem de resíduos recicláveis.

Com o desenvolvimento do SCOLIDSS, pretende-se auxiliar o planejamento e distribuição da coleta seletiva de resíduos sólidos, buscando a redução das distância percorridas pelos veículos de coleta e, também, a redução da quantidade de resíduos desperdiçados devido à falta de controle na capacidade de processamento de trabalho nas unidades de triagem. Observa-se na literatura sobre gestão de resíduos que as disposições finais consideradas pelos mesmos são geralmente ou o aterro sanitário, ou os incineradores, havendo uma desconsideração das unidades de triagem. O único artigo a tratar da disposição final de resíduos potencialmente recicláveis (Huang, 1998), o faz de maneira similar ao tratamento dado aos aterros sanitários, desconsiderando o fluxo dinâmico de entrada e saída de resíduos, característica das unidades de triagem de resíduos sólidos.

Para o desenvolvimento do sistema computacional apresentado no artigo foram utilizadas técnicas quantitativas oriundas da Pesquisa Operacional, tais como a simulação discreta e algoritmos para a resolução do roteamento de veículos. O uso destas técnicas, objetiva agregar qualidade ao processo decisório, pois muitas das vezes, as decisões sobre o planejamento da gestão dos resíduos sólidos são tomadas baseadas somente na experiência dos gestores (Chang, 1996). Fato este, que segundo este autor, contribui para o alto custo e o baixo desempenho dos sistemas de coleta de resíduos nas cidades. A utilização de ferramentas de Pesquisa Operacional (PO) na Gestão de Resíduos Sólidos surge como uma alternativa viável para o tratamento da complexidade inerente ao processo de coleta seletiva de resíduos sólidos, pois através do uso destas ferramentas pode-se representar uma situação do mundo real, estudar seu comportamento (via execução de modelos formais) e tomar decisões com base nas conclusões extraídas. Vários autores (Huang, 1998; Chang, 2000; Chung, 1996) já utilizaram técnicas e métodos da PO para desenvolver estudos na área de coleta de resíduos sólidos

O artigo está organizado da seguinte forma: na seção 2 é descrito o sistema de apoio à decisão - SCOLDSS, enfatizando-se a concepção da sua arquitetura. Na seção 3 são apresentados dados relativos à primeira etapa de validação do sistema e, por fim, na seção 4 são apresentadas as considerações finais do artigo. SCOLDS - O Siscisão SCOLDSS foi constura ditribuição dos resíduo

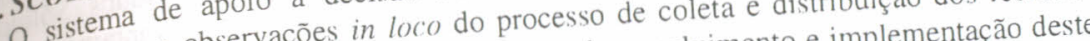

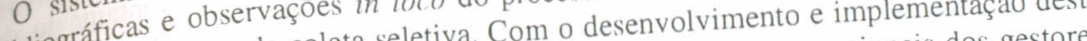
bibliog nenientes da coleta seletiva. Comada de decisões operacionals dos gestores sólidos provea-se subsidiar o processo de toma ne refere à logística dos resíduos sistema buscáduos sólidos (Goldbarg, 2000) no que se resíduos, nas unidades de da área de residuos coleta até a fase de entrega dos resícuos, nálise de possíveis

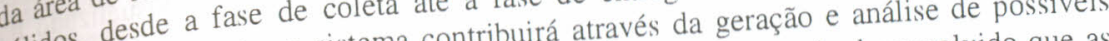
sólidos, Basicamente, o sistema contribuiá ansidera-se no estudo desenvolvido que as triagem. Basicane deste tipo de coleta. Consideras e

cenários de operaçantação da coleta seletiva (equipamentos, definidas.

periodicidade da coleta seletiva) já periodiclo para o desenvolvimento do SCOLDS Operacional (Law, 1991). O A metodo modelos em Pesquisa Operacionalorários, na qual o desenvolvimento de mode seguinte forma: (1) estudos exploratónios, na qual o desenvolvimento foi estruturado da seguinte (2) desenvolvimento da solução, pela desenvo foi identificado e estruturado, (2) denentema; (3) implementação problema modelos formais capazes de representar o problem a à decisão; (4) construção de modelos formis cando-se a tecnologia de sistemas de apoio àdecisão, (4) computacional da soluçă, util validaça da soluão, através de testes en laborvada. A validação fo valíaça do obtidos estão de acordo com a realidade déduos sólidos recicláveis resultados obtidos utilização de dados históricos da área de resín sos sóldosuisadores na desenvolvida com a utio Grande do Sul e, através da participação de pesquisadores na de un rea.

a de à Para o desenvolvimento do SCOLDSS foi utilizada a arquitetura de sistenas dremas básicos: decisão proposta por Sprague (1991), a qual é composta por apresentados nas próximas banco de dados, modelo decisório e interface, os qua na figura 1. A retuitetura do SCOLSS é apresentada na fig

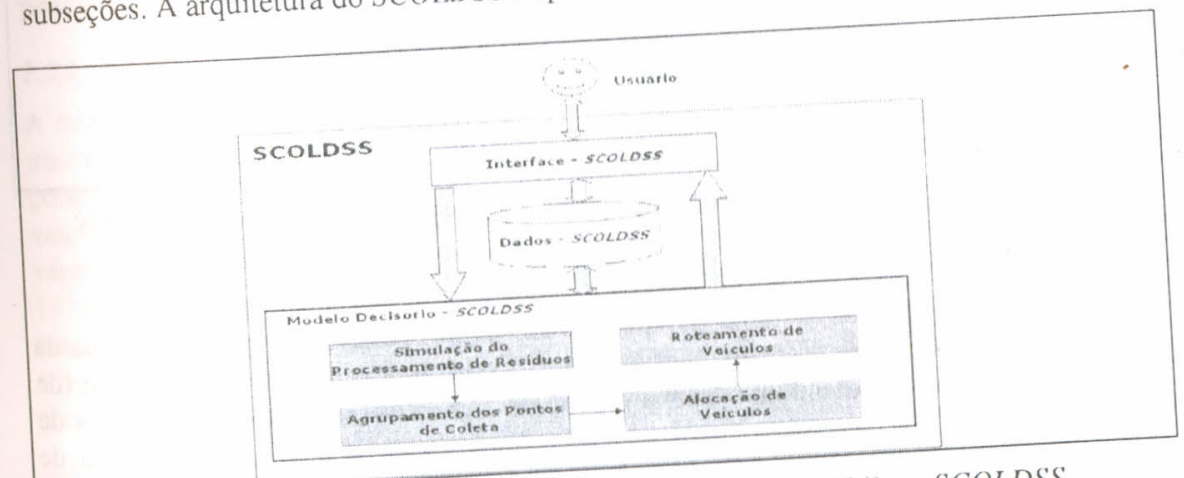

Figura 1 - Representação da arquitetura de Sprague (1991) no SCOLDSS

\section{1 - Subsistema banco de dados} a presceral importância à geração de informações para modelo, o qual trabalhará, basicamente, anco de dados utilizando os dados deste subsistema. Para o desenvolvimento do banc 
utilizados: trabalhos realizados anteriormente a este estudo (Huang 1998, Chung, 1996, Chang, 2000), manuais técnicos relativos à área de Gestão de Resíduos Sólidos (O'Leary, 1999; Monteiro, 2001), bem como de entrevistas para levantamento requisitos junto à especialistas em gestão de resíduos sólidos. Esta estrutura também fe utilizada como base de dados para o desenvolvimento do Sistema de Informação para a Coleta Seletiva de Resíduos (SICOLSE) (Simonetto, 2003). O diagrama entidade relacionamento do subsistema banco de dados do SCOLDSS pode ser visualizado figura 2.

\section{2 - Subsistema Modelo Decisório}

O subsistema modelo do SCOLDSS foi concebido utilizando-se duas técnicas distinta da Pesquisa Operacional: a simulação computacional de eventos discretos e o desenvolvimento de heurísticas para o problema do roteamento de veículos. A utilização destas duas técnicas é justificada pela natureza distinta dos problemas tratados, primeiro, a determinação da capacidade de processamento de resíduos e, segundo, a determinação do escoamento do fluxo de resíduos, em conseqüêência, do resultado das simulações. Baseado na integração da simulação do processamento de resíduos nas unidades de triagem, para a determinação da capacidade de processamento de resíduos em um dia e, a execução do problema do roteamento de veículos com múltiplo depósitos, serão determinados os percursos dos veículos de coleta de resíduos, bem como o destino final dos resíduos por eles transportados.

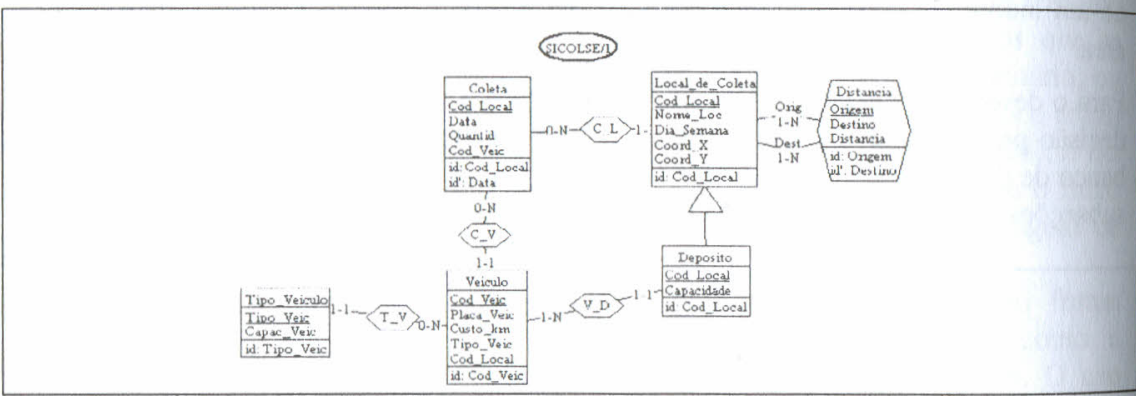

Figura 2- Diagrama entidade-relacionamento do modelo de dados do SCOLDSS

\subsection{1 - A Simulação de Eventos Discretos no SCOLDSS}

Quando da utilização do sistema de apoio à decisão, o usuário irá informar: o dia da semana para o qual será efetuado o planejamento da coleta seletiva, o mês do ano (de forma a considerar as eventuais sazonalidades existentes no processo); as unidades de triagem de resíduos operantes, para que seja determinada a quantidade máxima de matéria prima pós-consumo (em kg), que cada unidade em operação, é capaz de processar diariamente.

Nesta primeira etapa de utilização do modelo decisório executa-se um modelo de simulação computacional de eventos discretos, implementada no simulador Arena 3.5 para a determinação da capacidade de processamento de resíduos em cada unidade de triagem. Tal determinação faz-se necessária pela particularidade encontrada no caso de resíduos potencialmente recicláveis originada pelo fluxo de entrada e saída deste tipo de resíduo nas unidades de triagem. Ambos os fluxos não são verificados em se tratando reśduos sólidos que são direcionados ao aterro sanitario, pois neste tipo de dos resídua sõ existe a saída dos resíduos sólidos (somente a entrada de resíduos) disposição tinal, não ex, 1998; DMLU, 2004)

Chang, 2000; Huang, Nesta última etapa, tanto os resíduos selecionado en consideração pela Nesta última eno destino o aterro sanitario, têm de serem levados em consideraçáo pela que terẫo como destic ocupam espaćsico temporariamente e, também, consumiram simulação, pois ambos ocuparn trabalhadores. Para simular a simulaçaterminado tempo para serem processados pelos tramente em cada unidade de um determe máxima de resíduos a ser processada diariamente ente de resíduo sólido quantidade máa em consideração a média total coletada diariamente de resíduo sólido triagem é levada emićável, de acordo com o dia da semana e o mês do ano e, potencialmente reciclavel, de aco quilograma por minuto de trabalho em cada unidade. potenacidade de processamento em quilograma por minuto de trabantuais interrupções no Para tanto, considera-se também no mariação da produção de um turno trabalho (almoço, troca de equipe de trabalho) e a variação da prodsça de um turno trabalho (almo

listribuição dos resíduos sólidos durante a simulação foi utilizado o componente Para a distribuição dos residuos solidos dur enviar-se a matériaPickstation do simulador Arena 3.5, o qual seleciona a unidade parasos utilizados (de prima, em ordem de precedência, de acordo com: o número de recursos utilizados (de modo a evitar ociosidade) e, pela quantidade de matéria-pria fase do modelo decisório é a para ser processada. A informação gerada pela primeira fase do modelo decisón processar demanda de resíduos sólidos (em kg) que cada unidade de triagem é cã da simulação. demanda de rminado dia de trabalho, obtida pela média das $n$ execuções da simulação. em um determinado diassamento de resíduos em cada unidade de triagem é integrada ao A capacidade de processamento de restrição de capacidade ao problema do roteamento de modelo decisorio na formá de nenhuma veículos com vários de resíduos maior que a sua capidade de unidade de triagem recessamento simulada.

\section{2 - Agrupamento de Pontos de Coleta e Alocação de Veículos}

A fase subsequiente da utilização do modelo decisório caracteriza-se por possuirmos $n$ unide subequente da modelo) e $m$ unidades de triagem (com a demanda já definida pelácl a serem coletados pelos pontos de coleta com oferta de resíduo sólido reciclável a serem coléculos com veículos. Tal descrição denota claramente o problema do roteamento de veiculos (Gillet, vários depósitos, onde, para a resolução, foi utilizada a abordagem proposta por (Gillet, 1974), implementadas na forma de heurísticas do tipo "agrupar para depois rotear" Nesta abordagem, primeiramente devem-se associar pontos de coleta às unidades de triagem específicas. Ou seja, é executada uma determinação do tipo "os resíduos do ponto de coleta $x$ serão enviados para a unidade de triagem $y$ ".

Após o procedimento de agrupamento dos pontos de coleta, é efetuada a rotina de a implema forma que implementado no SCOLDSS o algoritmo simplex (Goldbarg, 2000), de un não se considerasse no modelo decisório cada veiculo individualmente, pois assim sua resolução poderia comprometer no aspecto tempo de resposta o desempenho do sistema como um todo. Para a resolução da alocação foram considerados os tipos de veículos, ou seja, os dived conforme as suasidades de carga. Com isto, consegue-se um processamento mais rápido do modelo, evitando possíveis explosões combinatórias no espaço de soluções. 
Como resultado final do processamento desta etapa tem-se estipulada a quantidade em quilogramas que um determinado tipo de veículo $k$ irá transportar para uma unidade triagem $i$. Porém, se a resolução envolver vários tipos distintos de veículos é necessário utilizar abordagens avançadas (metaheurísticas) para a resolução da alocação, tais como, a busca-tabu, algoritmos genéticos ou simulated annealing, pois o algoritmo simple comporta-se bem somente em problemas de pequeno e médio espectro (Cordeau, 2002).

\subsection{3 - Determinação do Roteiro da Coleta Seletiva}

Após a estimativa da quantidade máxima de processamento de resíduos por unidade; determinação do envio do resíduo coletado em cada ponto para uma unidade de triagem específica; da estipulação da quantidade de resíduo mínimo a chegar em cada unidada de triagem; e dos veículos alocados para execução da coleta, reduz-se o problema à configuração do problema de roteamento de veículos com um único depósito (Cordeau 2002). Como cada depósito (unidade de triagem) já possui uma determinação de quais os pontos de coleta the enviarão material para processamento, é necessário somente estabelecer a ordem que cada ponto de coleta será visitado. Para se garantir bons resultados nesta etapa, optou-se por utilizar o algoritmo para roteamento de veículos proposto por Renaud (2002), o qual é aplicado a problemas com frota heterogênea de veículos e apresenta excelentes resultados com problemas reais de roteamento. Para a determinação da oferta de resíduo em cada ponto de coleta, a qual é uma das restrições componentes do problema de roteamento de veículos, é utilizada a média de coleta (em $\mathrm{kg}$ ) no ponto de coleta. A quantidade média é estimada conforme o dia e o mês de coleta.

Nesta etapa, o objetivo é a geração das rotas de coleta a serem percorridas, bem como as atribuições de qual veículo deve percorrer cada rota. Após o processamento das rotinas é gerada uma resposta com a seguinte estrutura: para a unidade de triagem $x, 0$ veículo $n$ irá percorrer as pontos de coleta $a, b$ e c (nesta ordem). No SCOLDSS, o resultado é apresentado na forma de rotas em um relatório gráfico com mapa ilustrativo dos locais a serem percorridos, de modo a facilitar a comunicação com os usuários.

\section{3 - Subsistema Interface}

Para o desenvolvimento do subsistema interface do SCOLDSS foi levada em consideração a amigabilidade (user-friendly) da mesma para com os possíveis usuários finais, os gestores da área de resíduos sólidos, os quais não possuem a obrigação de serem especialistas na área computacional. Exemplo da interface principal do SCOLDSS pode ser visualizado na figura 3. A validação da interface foi desenvolvida com a participação de possíveis usuários (gestores e acadêmicos) do SCOLDSS.

\section{Validação do SCOLDSS}

O sistema SCOLDSS consiste do desenvolvimento de modelos quantitativos e de simulação para o planejamento operacional da coleta seletiva de resíduos sólidos. Como um modelo pode ser definido como "representação do mundo real" (Goldbarg, 2000) temos que fazer com que o comportamento da representação seja o mesmo (ou mais próximo possível) da realidade em questão, sob determinadas condições especificadas. A este processo denomina-se validação de modelo.

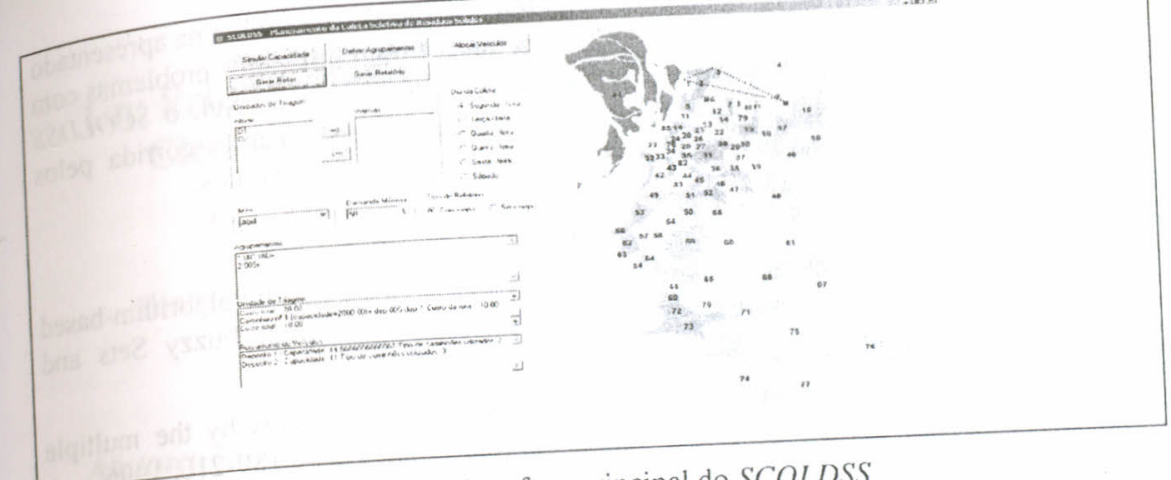

Figura 3 - Interface principal do SCOLDSS

palidação do módulo de simulação da capacidade de processamento de resíduos Para a validação do triagem foram utilizados dados reais de sete unidades de triagem. diário das unidades de triagen foram unse maneira correta, com um desvio médio de Nesta validação o sistema comportou-se de maneira correta, compenho do sistema, tendo $2.7 \%$ dos dados reais coletados,

em vista que a simulação trata da capacidade náxim bastante Na validação da alocação e do roteamento de veículos os resultados fora de resíduos significativos. Para esta validação foi utilizado um problema real com dimensões significativos. sólidos apresentado em Lanson (2002) apresentou melhorias aos resultado diminutas. Porém, o algoritmo de Renau (1999) utilizou a heurística savings e um apresentados. Para o roteamento, Larson (1999) gerado pela heurística. Em ambos os procedimento de melhoria do resultado (2-Opt) gerado pela hultados apresentados. Em casos, o algoritmo de Renaud (2002) foi superior aos resultados apresentem de cascentou resultados cor relação ao saving.s o algoritmo aprese $14.4 \%$ e, com relação ao procedimento de de $3.2 \%$.

resultados com custo mais baixos na ordem de $3.2 \%$. Utilizando-se dados reais da coleta seletiva, o SCOLDSS apresentou significatios de melhorias, reduzindo, em média, 8,82\% o total da distancia peral do número de viagens coleta, e também na redução de $17,89 \%$ (em média) do total do número de destes veículos.

\section{Considerações Finais}

artigo teve como objetivo principal apresentar um sistema de apoio à decisão (SCOLDSS) con a restores da área de réduos sólidos no (SCOLDSS) concebido para auxiliar gestores da ara a especificação dos planejamento operacional da coleta seletiva. Basicanente, para a especisa em artigos requisitos e desenvolvimento do mesmo for desenvolvida una pesquisa em anto científicos mo tanto do processo de coleta, qua cientificos relativos, observaçôes in teriormente, feita a validação processamento dos resíduos nas unidades de triagem e, posteriorménos de um município do sistem gaúcho.

Em uma primeira fase de validação do SCOLDSS, pôde-se constatar que o mesmo apresenta um comportamento compatível com a realidade, no c a resultados no roteamento de 
veículos, através da utilização do algoritmo de Renaud (2002), no problema apresentad por Larson (1999). Na segunda fase da validação, onde foram utilizados problemas com dados reais da coleta seletiva de um município do Rio Grande do Sul, o SCOLDS apresentou significativas melhoras, tanto na redução da distância percorrida pelos veículos coletores, como na redução do número de viagens destes veículos.

\section{Referências Bibliográficas}

Chang, N.; Wei, Y. Siting recycling drop-off in urban area by genetic algorithm-base fuzzy multiobjective nonlinear integer programming modeling. Fuzzy Sets and Systems, 114, pp.133-149, 2000

Chung, S.S.; Poon, C.S. Evaluating waste management alternatives by the multiple criteria approach. Resources, Conservation and Recycling, 17, pp.189-210, 1996.

Cordeau, J.F.; et al. A guide to vehicle routing heuristics. Journal of the Operational Research Society, 53, pp.512-522, 2002.

Departamento Municipal de Limpeza Urbana de Porto Alegre. In htup://www. portoalegre.rs. gov. br/dmlu/coletas.htm. Acessado em 10/02/2004

Fisher, M.; Jaikumar, R. A generalized assignment heuristics for vehicle routing. Networks, 11, pp. 109-124, 1981

Gillet, B.; Miller, L.R. A heuristic algorithm for the vehicle dispatch problem Operations Research, 22, pp.340-349, 1974.

Goldbarg, M.C.; Pacca Luna, H. Otimização combinatória e programação linear Campus, 2000.

Huang, G.H.; Baetz, B.W.; Patry, G.G. Trash-Flow Allocation: Planning Under Uncertainty. Interfaces, Vol. 28, No. 6, pp. 36-55, Nov-Dec, 1998

Larson, R.C.: Odoni, A.R. Urban Operations Research. Massachusetts Institute of Technology, 1999. Disponível na Web em: http://web.mit.edu/urban_or__book/www/book/

Law, A.M., Kelton, W.D. Simulation Modeling \& Analysis. 2 "Ed., McGraw-Hill, 1991.

Monteiro, J.H.P.; et al. Manual de Gerenciamento Integrado de Resíduos Sólidos. Rio de Janeiro: Instituto Brasileiro de Administração Municipal, 2001.

O'Leary, P.R; et al.. Decision Maker's Guide to Solid Waste Management.Vol. 2. Washington DC: U.S. Environmental Protection Agency, 1999.

Renaud, J: Boctor, F.F. A sweep-based algorithm for the fleet size and mix vehicle routing problem. European Journal of Operational Research, 140, pp. 618-628, 2002

Simonetto, E.O.;Borenstein, D.;Dotto, B.R. SICOLSE - Um Sistema de Informação para à Gestão da Coleta Seletiva de Resíduos. Anais do XXIII Encontro Nacional de Engenharia de Produção. UFOP-ABEPRO, Ouro Preto-MG, Outubro, 2003.

Sprague, R.; Watson, H. Sistemas de Apoio à Decisão: Colocando a Teoria em Prática. Rio de Janeiro, Campus, 1991 